\title{
CONSTRUÇÃO E VALIDAÇÃO DA CURVA DE CALIBRAÇÃO EM NIR ATRAVÉS DE ANÁLISES BROMATOLÓGICAS DA POLPA DE GOIABA
}

\author{
Jéssika Santos de Oliveira ${ }^{1}$ \\ Mylena Gonçalves Raymundo² \\ Sara Maria Andrade Pereira ${ }^{3}$ \\ Adriano Azevedo Merçon ${ }^{4}$ \\ Jaqueline Rodrigues Cindra de Lima Souza ${ }^{5}$ \\ Tércio da Silva de Souza ${ }^{6}$ \\ Adésio Ferreira ${ }^{7}$
}

Resumo: A P guajava L, comumente conhecida como goiaba, ocorre naturalmente em todo território brasileiro, sendo mais explorada na região nordeste e sudeste do país. O mercado brasileiro de goiaba está dividido em dois segmentos: o de goiaba in natura e o de goiaba para indústria. A avaliação da qualidade dos frutos é uma questão importante, porém, as metodologias convencionais utilizadas necessitam de extração prévia dos analitos. A espectroscopia na região do infravermelho é uma ferramenta com alto potencial analítico, pois fornece o perfil completo da composição de uma amostra em poucos segundos, apresenta alta sensibilidade, é de fácil implementação e baixo custo.

Palavras-chave: Goiabeiras; Genótipo; Bromatologia; NIR.

\footnotetext{
1 Instituto Federal do Espírito Santo, Brasil. E-mail: jessikaoliveira664@gmail.com.

2 Instituto Federal do Espírito Santo, Brasil. E-mail: mylenagr@hotmail.com.

3 Instituto Federal do Espírito Santo, Brasil. E-mail: saramape@yahoo.com.br.

${ }^{4}$ Instituto Federal do Espírito Santo, Brasil. E-mail: adriano.azevedo@ifes.edu.br.

${ }^{5}$ Instituto Federal do Espírito Santo, Brasil. E-mail: jrsouza@ifes.edu.br.

6 Instituto Federal do Espírito Santo, Brasil. E-mail: tssouza@ifes.edu.br.

7 Instituto Federal do Espírito Santo, Brasil. E-mail: adesioferreira@gmail.com.
} 